\title{
Numerical Investigation of Electro-magnetic Flow Control Phenomenon in a Tundish
}

\author{
Anurag TRIPATHI \\ Research \& Development, Tata Steel, Jamshedpur-831001, India. E-mail: anurag.tripathi@tatasteel.com \\ (Received on October 3, 2011; accepted on November 10, 2011)
}

\begin{abstract}
The liquid steel flow phenomenon in a tundish is important to improve the steel quality. It is governed by the design of the tundish and its flow control devices. Pouring chamber is the most popular flow control device used inside the tundish. Flow control using electro-magnetic forces is the latest development in this area. The present work investigates the electromagnetic flow control phenomenon in the tundish. A 3-D Magnetohydrodynamics (MHD) model was used to study the flow behavior governed by electromagnetic forces in the tundish. The innovative concept of using electromagnetic dam as a flow modifier for tundish was proposed in current work. Computational Fluid Dynamics (CFD) simulations were performed for different combinations of electromagnetic dams and results were compared with pouring chamber. The advantage of electromagnetic flow control over other mechanical devices was established through CFD simulations.
\end{abstract}

KEY WORDS: tundish; plug volume; dead volume; mean residence time; inclusion flotation; electromagnetic dam.

\section{Introduction}

The continuous improvement in cleanliness of steel is an important parameter to increase the steel demand in the market. The inclusion separation from molten steel in the tundish is a way to achieve superior steel quality. Tundish is a metallurgical vessel, which act as a buffer during continuous casting. It is used to refine the steel through inclusion flotation. Inclusion flotation depends upon liquid steel flow behaviour in the tundish. The RTD characteristic of flow pattern is an established criterion for predicting the inclusion separation efficiency of the tundish. Researchers have established certain RTD characteristics for achieving the maximum inclusion separation ratio. The flow control is required to achieve the desired RTD characteristics in the tundish. The traditional flow control devices used inside the tundish are dams and weirs. Dams and weirs have improved the flow characteristics, but resulted in a reduction of effective volume. Pouring chamber is the most popular flow modifier used in the modern steel industries. The pouring chamber is used to suppress the flow near the pouring region of the tundish. RD. Morales et al. have investigated the role of pouring chamber to suppress the turbulence in the inlet stream. ${ }^{1)}$

The design of an effective flow control mechanism is still a challenge. The understanding of flow phenomenon inside the tundish is pre-requisite to design flow control device. Researchers have reported various investigations on fluid flow phenomenon inside the tundish. Investigators claim that the suppression of flow in the pouring region will enhance the inclusion flotation characteristics. Tripathi and
Ajmani have shown that the development of the rotatory flow near the pouring region enhances the plug volume.2) The investigations to generate the desired flow pattern through effective flow control mechanism are of interest to the scientific community.

The use of electromagnetic forces for controlling the flow in the tundish is the latest development in flow control technology. Investigators have shown the strong impact of electromagnetic flow control system to produce the desired flow pattern in the tundish. ${ }^{3,4)}$ However, the design of such system is a complicated process. The complete understanding of flow phenomenon guided by electromagnetic forces is required to design this system for tundish. Researchers have reported various investigations done to understand the flow behaviour driven by electromagnetic forces. ${ }^{5-8)}$ Votyakov et $a l$. have used magnetic obstacle in flow field to understand the complexity of electromagnetic flow control phenomenon. ${ }^{9)}$ Investigators such as Man et al. have successfully simulated the electromagnetic flow control process for mold. ${ }^{10)}$ The challenge to design an effective electromagnetic system inspired us to investigate the electromagnetic flow control mechanism in a tundish.

The investigations were performed in the present work to understand the flow phenomenon governed by electromagnetic forces in the tundish. Our investigation results in the development of an innovative electromagnetic flow control device for tundish. This device will work as a dam, without the presence of any physical device inside the tundish. Hence, the device was named as an electromagnetic dam and its comparative analysis was performed with pouring chamber in the present work. 


\section{Model Development}

\subsection{Geometrical Description}

The current work is an investigation about the role of electromagnetic dams in improving the flow characteristics in the tundish. Table 1 shows the set of simulations performed for present work. The electromagnetic dams used for current investigation can be seen from different views of tundish. Figs. 1(a), 1(b) and 1(c) presents the top view of the symmetrical half at different heights of tundish. The location of electromagnetic dams with the direction of imposed magnetic field can be identified from these figures. The region of the imposed magnetic field can be further seen from the vertical sectional view of the tundish in Fig. 1(d).

(a)

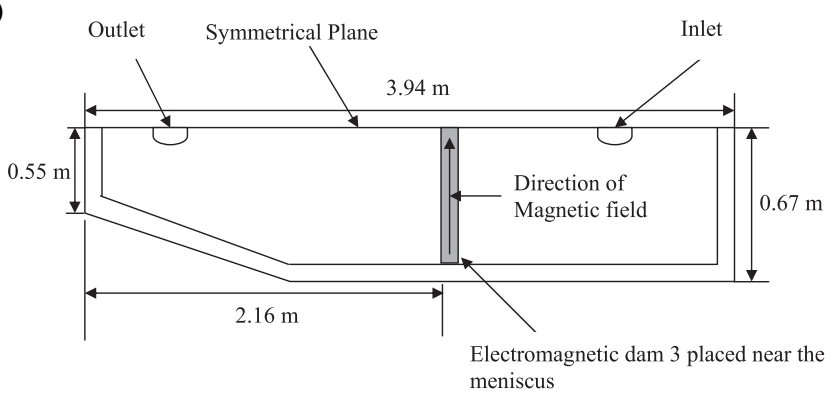

(b)

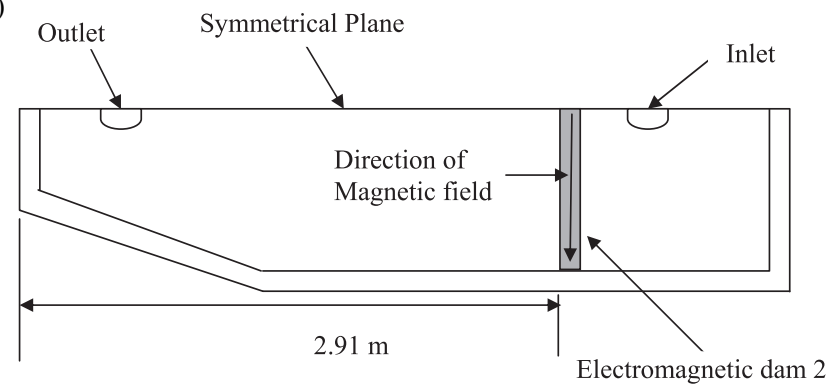

(c)

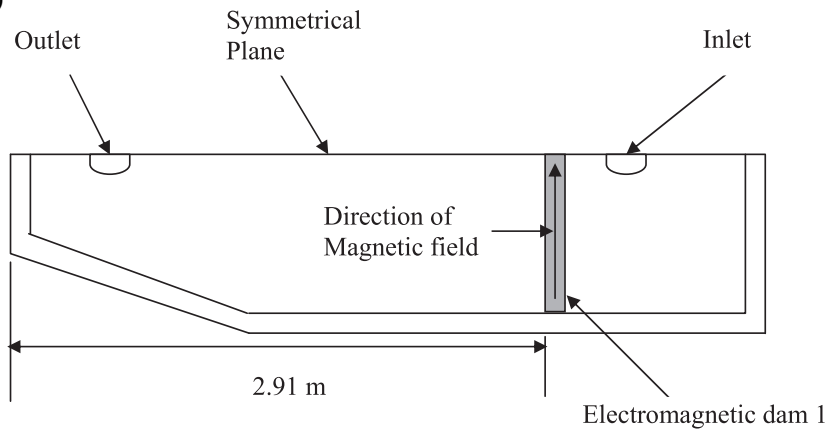

(d)

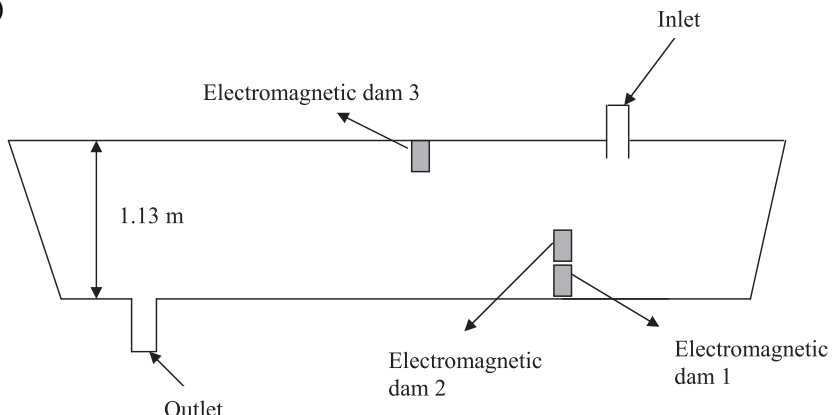

Table 1. No. of cases chosen for simulation.

\begin{tabular}{ccccc}
\hline S. No & $\begin{array}{c}\text { Pouring } \\
\text { Chamber }\end{array}$ & $\begin{array}{c}\text { Electromagnetic } \\
\text { Dam 1 } \\
\text { (magnetic } \\
\text { strength) }\end{array}$ & $\begin{array}{c}\text { Electromagnetic } \\
\text { Dam 2 } \\
\text { (magnetic } \\
\text { strength) }\end{array}$ & $\begin{array}{c}\text { Electromagnetic } \\
\text { Dam 3 } \\
\text { (magnetic } \\
\text { strength) }\end{array}$ \\
\hline Case 1 & Not used & Not Used & Not Used & Not Used \\
Case 2 & Not used & $0.1 \mathrm{~T}$ & Not Used & Not Used \\
Case 3 & Not Used & $0.15 \mathrm{~T}$ & Not Used & Not Used \\
Case 4 & Not Used & $0.2 \mathrm{~T}$ & Not Used & Not Used \\
Case 5 & Not Used & $0.25 \mathrm{~T}$ & Not Used & Not Used \\
Case 6 & Not Used & $0.3 \mathrm{~T}$ & Not Used & Not Used \\
Case 7 & Not Used & $0.25 \mathrm{~T}$ & $-0.27 \mathrm{~T}$ & Not Used \\
Case 8 & Not Used & $0.25 \mathrm{~T}$ & $-0.27 \mathrm{~T}$ & $0.15 \mathrm{~T}$ \\
Case 9 & Used & Not Used & Not Used & Not Used
\end{tabular}

(e)
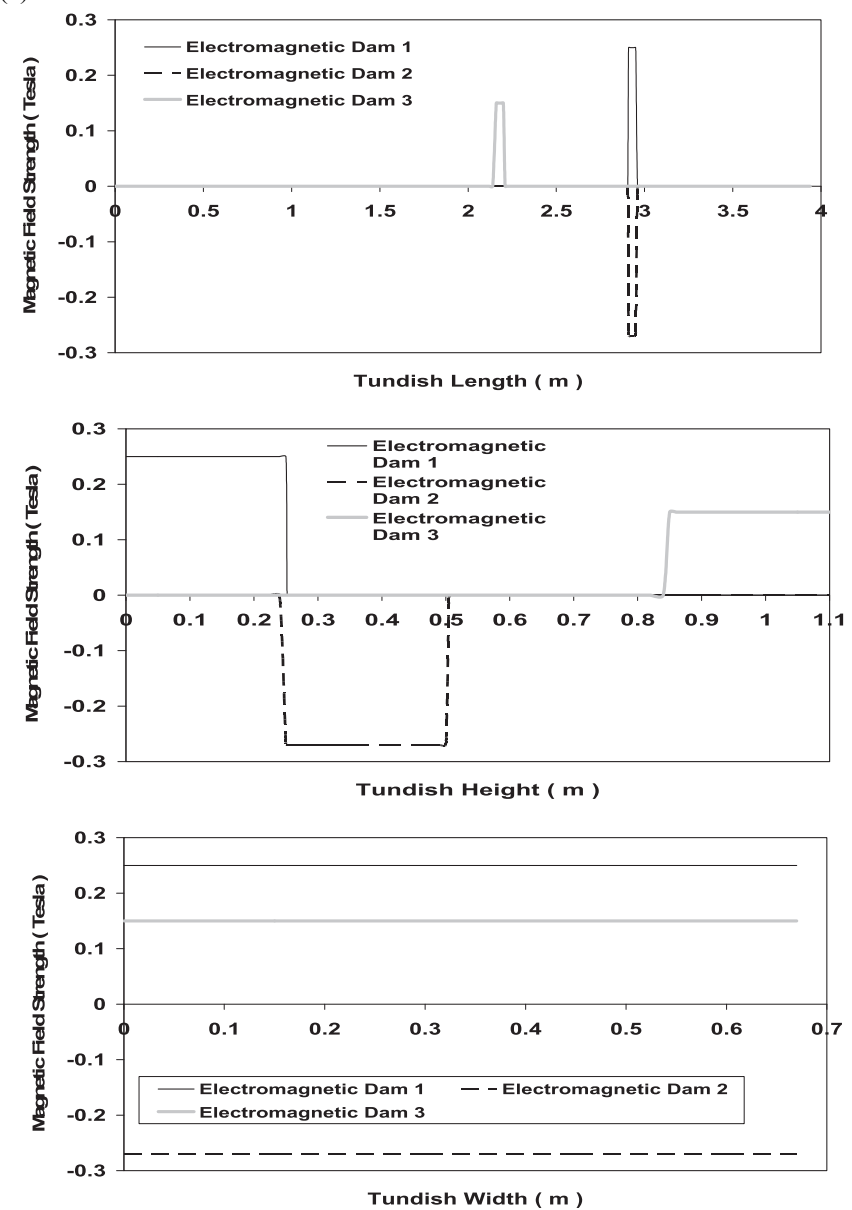

Fig. 1. (a) Top view of symmetrical half of tundish at horizontal plane ( $\mathrm{Y}=1.13 \mathrm{~m}$ ) with proposed electromagnetic dam. (b) Top view of symmetrical half of tundish at horizontal plane $(\mathrm{Y}=0.25 \mathrm{~m}$ ) with proposed electromagnetic dam. (c) Top view of symmetrical half of tundish at horizontal bottom plane $(\mathrm{Y}=0)$ with proposed electromagnetic dam. (d) Vertical sectional view of the proposed invention at symmetrical plane with marked region of imposed magnetic field. (e) Applied Magnetic field strength with regard to tundish dimensions for Case 8 . 
The electromagnetic dams are numbered as 1, 2 and 3 in Fig. 1(d). The height of the magnetic filed region is $25 \%$ of the tundish bath height and thickness is $50 \mathrm{~mm}$. The magnetic field zones marked in figures are perpendicular to the principal direction of the molten steel flow from the inlet to outlet portion. The simulations were performed for symmetrical half of the tundish. Fig. 1(e) presents the graphical representation of the applied magnetic field. Fig. 1(e) represents the case 8 of Table 1 . The dimensions of the pouring chamber simulated for comparative analysis with electromagnetic dam can be seen from Figs. 2(a) and 2(b). Table 2 shows the operating parameters for current investigation.

\subsection{Governing Equations}

The continuity and momentum conservation equation in three-dimensional was solved to compute the flow field in the tundish. The details of the model equation are already published in our previous paper. ${ }^{4}$ The model assumes that magnetic effect of electromagnetic dam is confined only at the position of dams. The rest of the domain is not affect by the applied magnetic field. The reason behind this assumption is a low Magnetic Reynolds Number $\left(\operatorname{Re}_{\mathrm{m}}=L_{\infty} U_{\infty} \mu_{\infty}\right.$ $\sigma$ ) for liquid steel and uniform applied magnetic field. The induced magnetic field is very small as compared to applied magnetic field for $\operatorname{Re}_{\mathrm{m}}<<1$. Hence, when applied magnetic

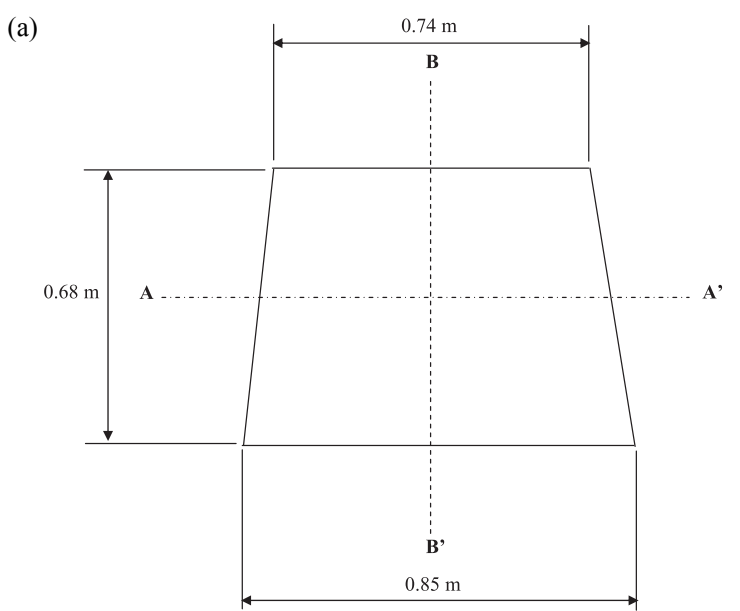

(b)

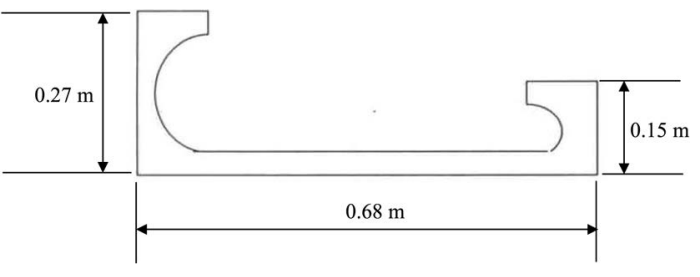

Fig. 2. (a) Top view of the pouring chamber used for carrying out simulation for the tundish. (b) View of the pouring chamber for section BB'.

Table 2. Operating parameters used for simulation.

\begin{tabular}{ccc}
\hline S. No. & Parameter & Values \\
\hline 1 & Bath height $(\mathrm{m})$ & 1.13 \\
2 & Submergence depth of shroud $(\mathrm{m})$ & 0.35 \\
3 & Shroud diameter $(\mathrm{m})$ & 0.085 \\
4 & Outlet nozzle diameter & 0.081 \\
5 & Throughput (ton/min) & 3.5 \\
\hline
\end{tabular}

field is constant with regard to time and $\mathrm{Re}_{\mathrm{m}}<<1$, the variation of magnetic field with time can be neglected. Therefore, the present model incorporates the Lorentz force as a source in the momentum equation, only at the location of electromagnetic dams. Table 3 shows the expression for the RTD characteristics.

\subsection{Boundary Conditions}

Inlet velocity of $1.45 \mathrm{~m} / \mathrm{s}$ was set for the incoming jet with a turbulent intensity of $2 \%$. The rest of the boundary conditions are similar to the one used in our previously published papers. ${ }^{2,4)}$

\subsection{Numerical Procedure}

The computational domain of the half tundish was prepared and divided into small cells through gambit software. The numerical methodology adopted is similar to the one published in our earlier papers. ${ }^{2,4)}$ The commercial Computational fluid dynamics (CFD) package (FLUENT) was used to solve the discretized equation.

\section{Model Validation}

The experimental validation of the computational method was established with the experiment data reported in the literature. The matching between experiment and computation was already reported in our previous papers. ${ }^{2,4)}$ Investigators have reported the validation of Magnetohydrodynamics (MHD) equation with analytical solution of Hartmann problem. ${ }^{11,12)}$ The same methodology was adopted to validate the MHD model used in current work and is already published.

Hartmann flow is a steady flow of an electrically conducting viscous fluid between parallel non conducting channels with an applied transverse magnetic field. The governing equation for this problem is similar to the model equation used in current work. The inlet velocity for this problem was kept at $2 \mathrm{~m} / \mathrm{s}$. The simulation was performed for certain range of Hartmann no. from 0 to 25 . Hartmann no. is defined as the ratio of electromagnetic forces to non electromagnetic forces. The increase in Hartmann no. represents the rise in strength of electromagnetic forces. The simulation results of Hartmann flow were compared with the analytical solution of this problem. ${ }^{11,12)}$ Figure 3(a) shows the velocity profile computed from simulation results with increasing Hartmann number. The similar type of trend in the velocity profile computed through analytical expression was reported in the literature. ${ }^{11,12)}$ The comparison of predicted velocity profile with the one computed from analytical expression for Hartmann problem can be seen from Fig. 3(b).

Table 3. Expressions for RTD Characteristics.

\begin{tabular}{ccc}
\hline S.No & RTD Characteristics & Expressions \\
\hline 1 & Actual mean residence time $\left(\mathrm{t}_{\mathrm{m}}\right)$ & $\mathrm{t}_{\mathrm{m}}=\frac{\sum C_{a v} t \Delta t}{\sum C_{a v} \Delta t}$ \\
2 & Fraction of plug volume $\left(\mathrm{V}_{\mathrm{PV}}\right)$ & $\mathrm{V}_{\mathrm{PV}}=\mathrm{t}_{\mathrm{min}} / \mathrm{t}_{\mathrm{r}}$ \\
3 & Fraction of dead volume $\left(\mathrm{V}_{\mathrm{DV}}\right)$ & $\mathrm{V}_{\mathrm{DV}}=1-\mathrm{t}_{\mathrm{m}} / \mathrm{t}_{\mathrm{r}}$ \\
4 & Fraction of mixed volume $\left(\mathrm{V}_{\mathrm{MV}}\right)$ & $\mathrm{V}_{\mathrm{MV}}=1-\mathrm{V}_{\mathrm{PV}}-\mathrm{V}_{\mathrm{DV}}$ \\
\hline
\end{tabular}




\section{Results and Discussion}

The 3-D MHD simulation was performed for the tundish with electromagnetic dam of varied magnetic field strengths. CFD simulation was also performed for the tundish by increasing the number of electromagnetic dams. The performance of tundish using electromagnetic dams was compared with tundish having pouring chamber as flow

(a)

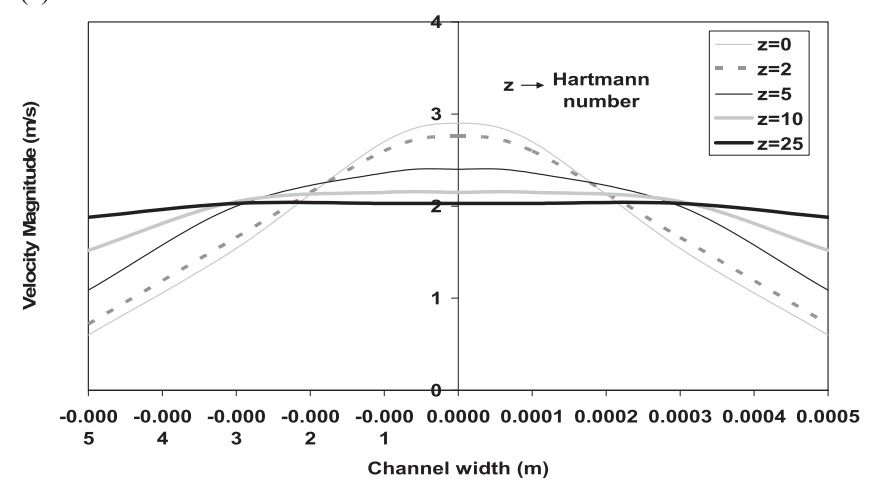

(b)

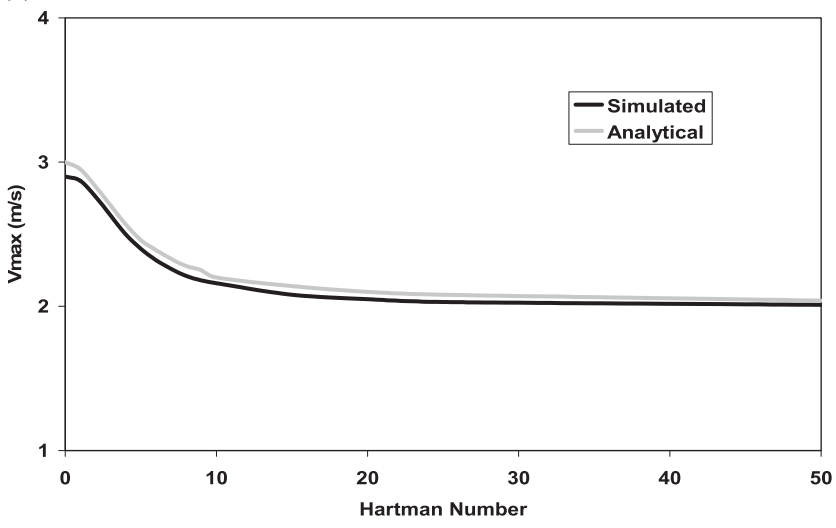

Fig. 3. (a) Velocity profile for Hartmann flow at different Hartmann numbers. (b) Comparison of the simulated versus analytical velocity at different Hartmann number. control device. The results obtained from the simulation will be presented in the subsequent steps.

\subsection{Fluid Flow Phenomenon in a Tundish with Electro- magnetic Dam}

The fluid flow analysis was performed for tundish embedded with electromagnetic dams. The electromagnetic dam 1 shown in Figs. 1(c) and 1(d) was initially used for understanding the flow behaviour inside the tundish. Electromagnetic dam 2 and 3 were then used to generate the superior flow characteristics. The flow patterns generated using only electromagnetic dam 1 can be seen from Figs. 4 and 5. Figure 4 shows the flow patterns at vertical symmetrical plane $(Z=0)$ for tundish with electromagnetic dam 1 and bare tundish. The surface directed flow vector can be seen for electromagnetic dam as compared to downward directed flow for bare tundish in Fig. 4. However, the intensity of the flow vectors was significantly high for flow patterns generated using electromagnetic dams. The recirculatory flow generated near the pouring region for electromagnetic dam can be noticed from Fig. 4. This recirculatory flow will enhance the mixed volume and will lead to reduction in dead volume. The flow behaviour can be further analysed by observing the flow pattern at the meniscus in Fig. 5. The high magnitude of the flow vectors for electromagnetic dam can be again noticed from Fig. 5. The high velocity at the meniscus is extremely undesirable as it can lead to reverse flow and formation of small circulation cell. These small circulation cells can be seen for the case of electromagnetic dam in Fig. 5. The flow pattern generated for electromagnetic dam was found to have some quality of desired flow characteristics, but high magnitude of velocity was its major disadvantage.

\subsection{Effect of Magnetic Strength of Electromagnetic Dam on Flow Patterns in Tundish}

The magnitude of applied electromagnetic braking force is critical for generating the desired flow pattern in the

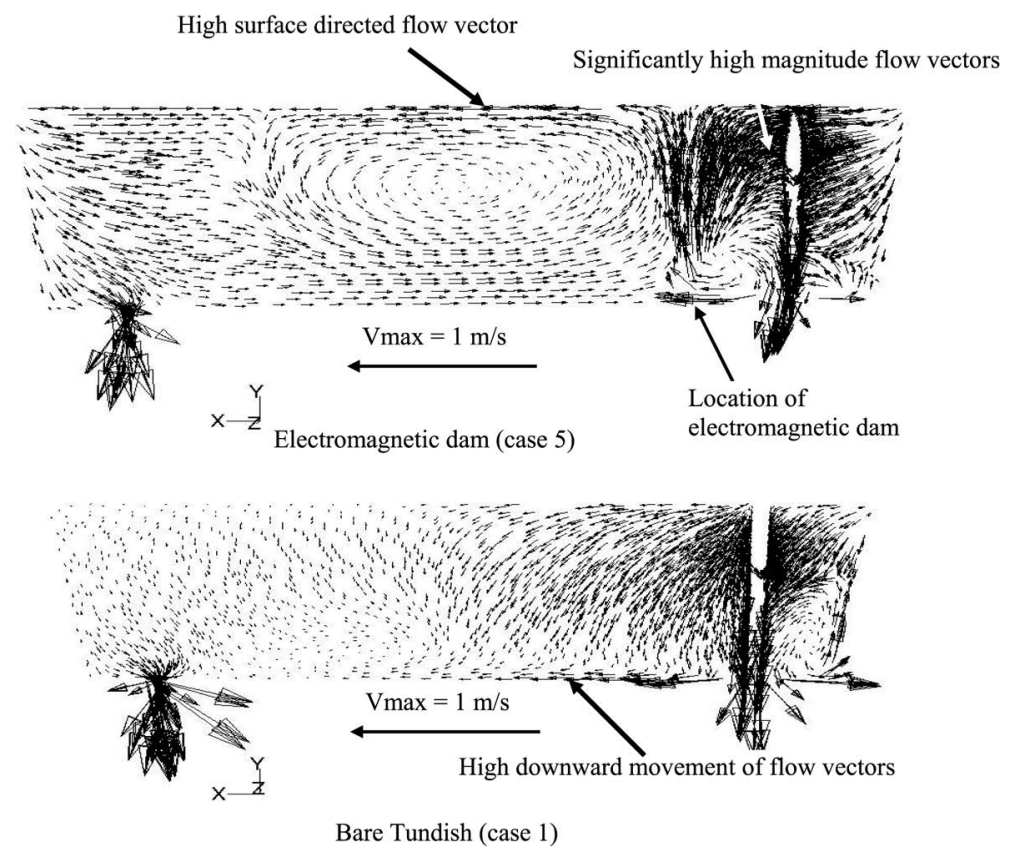

Fig. 4. Flow patterns at symmetrical vertical plane for tundish with single electromagnetic dam of case 5 and bare tundish. 
ISIJ International, Vol. 52 (2012), No. 3

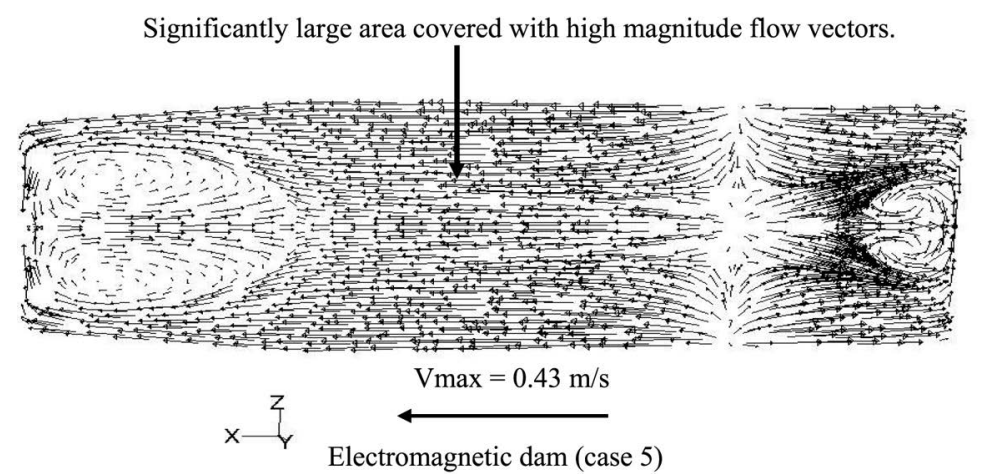

High magnitude flow vectors confined to smaller region
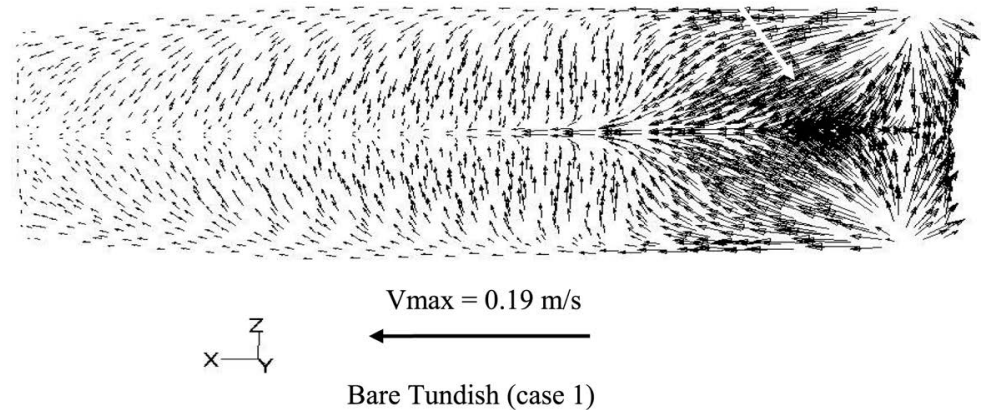

Fig. 5. Flow patterns at meniscus for tundish with single electromagnetic dam of case 5 and bare tundish.

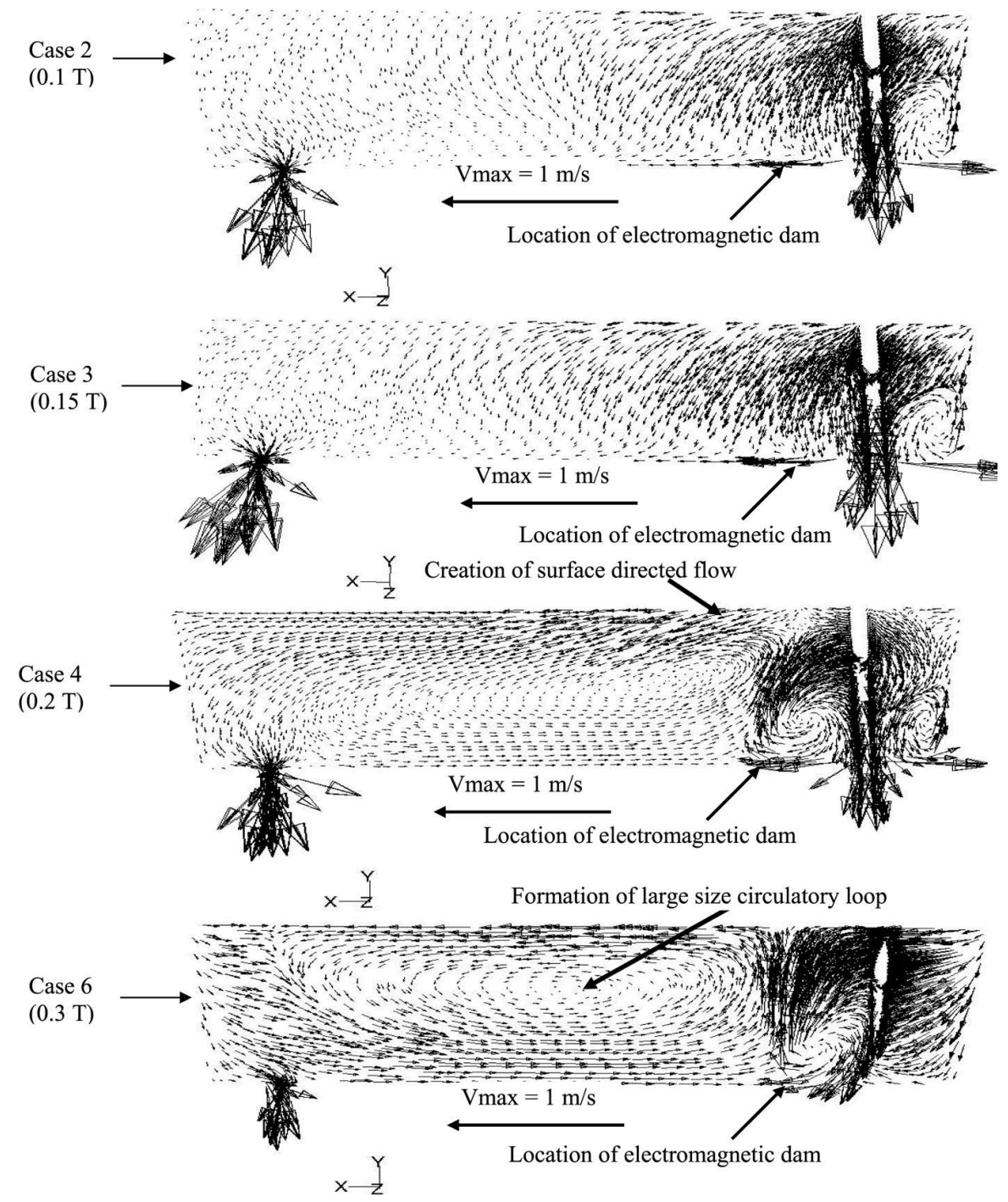

Fig. 6. Flow patterns at symmetrical vertical plane for tundish with single electromagnetic dam of different magnetic strengths. 
tundish. The magnitude of this braking force depends upon strength of applied magnetic field. Hence, it was felt that role of magnetic strength of electromagnetic dam needs to be investigated. The simulation was performed for tundish with electromagnetic dam 1 of different magnetic strength. Case 2 to case 6 of Table 1 shows the details of simulation performed for this study.

Figure 6 shows the flow pattern at vertical symmetrical plane $(Z=0)$ for different strength of electromagnetic dam. The flow pattern for magnetic strength of 0.1 and 0.15 tesla looks similar to the one obtained for bare tundish. However, the reduction in the downward movement of the flow vectors for tundish fitted with electromagnetic dam can be noticed. The reduction in downward motion of molten steel is due to decrease in magnitude of flow velocity near electromagnetic dam. It can be noticed from Fig. 6 that further increase in the magnetic strength results in reversal of flow vectors towards the surface.

The increase in magnitude of the velocity vector at high magnetic strength can also be seen from Fig. 6. The surface directed flow will strengthen the inclusion flotation but increase in velocity magnitude beyond optimum level is det- rimental for tundish.

Figure 7 shows the comparison of flow pattern at the meniscus for different magnetic strength. The increase in velocity magnitude with increase in magnetic strength can be noticed from Fig. 7. The high velocity at the meniscus can lead to formation of small circulation cell and thus expand the region of dead volume. The formation of circulation cells at high magnetic strength of 0.2 and 0.3 tesla can be observed from Fig. 7.

\subsection{Flow Patterns for the Tundish with Combination of Two Electromagnetic Dam}

The surface directed flow field generated at high magnetic strength had large magnitude of velocity vectors. The suppression of velocity magnitude was desired to take advantage of flow field generated by electromagnetic dam of high magnetic strength. Hence, simulation was performed with combination of two electromagnetic dams. The electromagnetic dam 1 and electromagnetic dam 2 as shown in Fig. 1(d) was used for the simulation. The opposite direction of magnetic field for electromagnetic dam 1 and 2 can be visualised from Figs. 1(b) and 1(c). The electromagnetic dam 1
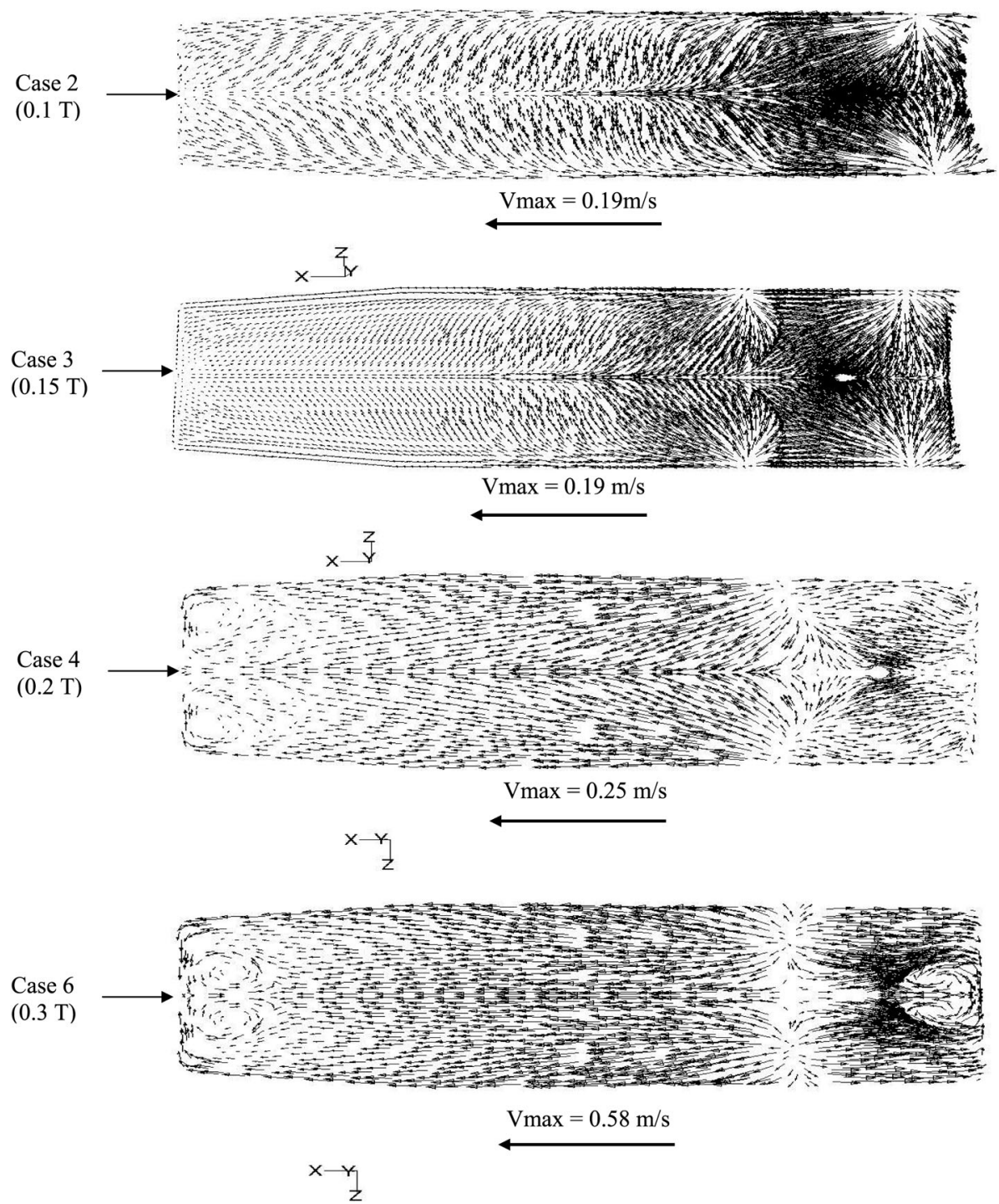

Fig. 7. Flow patterns at meniscus for tundish with single electromagnetic dam of different magnetic strengths. 
at magnetic strength above 0.15 tesla will reverse the flow and direct it towards the surface. Hence, magnetic field for electromagnetic dam 2 is applied in direction opposite to that of electromagnetic dam 1 . This magnetic field of electromagnetic dam 2 will generate the braking force for flow vectors moving towards the surface. Thus, the application of electromagnetic dam 2 will reduce the magnitude of the flow velocity.

The case 7 presented in Table 1 was simulated to investigate the effect of using two electromagnetic dam. Figure 8(a) shows the flow pattern at symmetrical vertical plane $(Z=0)$ for case 7 . The flow vectors in this plane move towards the surface and then rotate near the shroud. This development of rotatory flow near the shroud will enhance the mixed volume and reduce the dead volume. The flow intensity appears to be reduced as compared to one observed for case 4 to 6 . However, the magnitude of the flow velocity was quite high near the outlet. The flow velocity near the outlet needs to be reduced to optimum level for enhancement of plug volume. The flow field for vertical $\mathrm{YZ}$ plane at the centre of the shroud $(X=3.4)$ can be seen from Fig. 8 (b). The incoming jet of flow vector strikes the tundish bottom and develops the clockwise recirculatory flow at this plane. The flow velocity was found to be high toward the bottom and low at the top. Figure 8(c) shows the velocity field at the meniscus for case 7. The magnitude of the flow velocity appears to be non uniform and still high at the meniscus. The maximum magnitude of velocity was observed at the centre of the meniscus. Hence, the intense flow was created at the centre of the meniscus. The circulatory cells can be seen at all the corners in Fig. 8(c). Hence, meniscus velocity for case 7 was still above the desired optimum level. Thus, the meniscus velocity needs to be reduced for achieving superior performance as compared to pouring chamber.

\subsection{Comparison of Electromagnetic Dam with Pouring Chamber}

The pouring chamber is the best known flow control device used in the industry. It was felt that the commercial application of electromagnetic dam can not be justified without comparing its performance with the pouring chamber. The study performed for case 7 shows the improvement in the flow pattern, but still it is not competitive enough to replace the pouring chamber. The high magnitude of the meniscus velocity was the major disadvantage with the flow field generated for case 7 . Hence, the electromagnetic dam 3 was placed in the tundish to reduce the meniscus velocity. The location of electromagnetic dam 3 can be seen from Fig. 1(d). The case 8 presented in Table 1 was simulated to establish the superiority of electromagnetic dam over pouring chamber. The magnetic strength of electromagnetic dam 3 in case 8 was comparatively lower as compared to electromagnetic dam 1 and 2 . The flow pattern and Residence time distribution (RTD) performance for case 8 was compared with the flow field and RTD data generated for pouring chamber. Case 9 in Table 1 shows the study performed for pouring chamber.

Figure 9 shows the comparison of the flow pattern for pouring chamber and electromagnetic dam of case 8 at vertical symmetrical $X Y$ plane $(Z=0)$. The flow velocity for pouring chamber was found to be high in the pouring region and near the surface. It was comparatively low in rest of the (a)

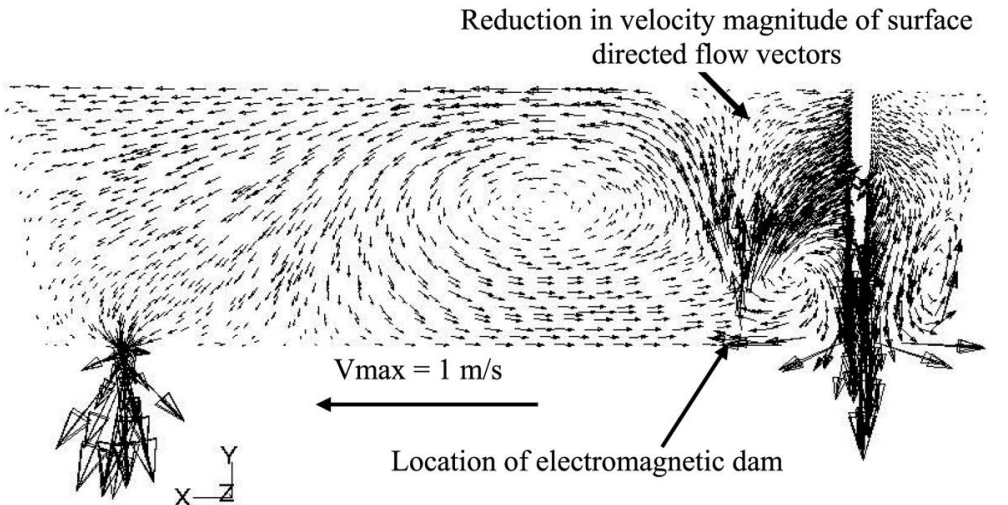

(c)

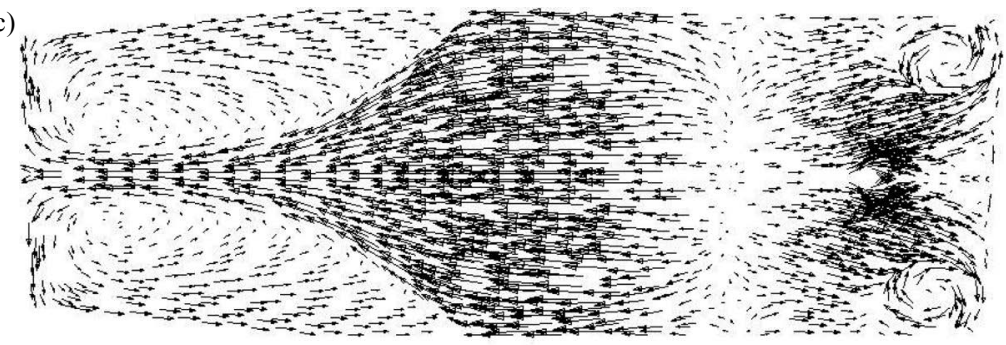

(b)

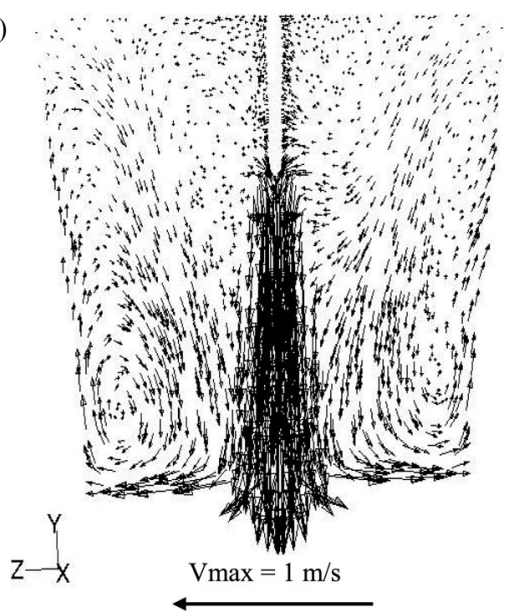

$\mathrm{V} \max =0.16 \mathrm{~m} / \mathrm{s}$

$X \frac{Y}{Z}$

Fig. 8. (a) Flow pattern at symmetrical vertical plane for tundish with two electromagnetic dam of case 7. (b) Flow pattern at vertical $\mathrm{YZ}(\mathrm{X}=3.4)$ plane for tundish with two electromagnetic dam of case 7. (c) Flow pattern at meniscus for tundish with two electromagnetic dam of case 7. 


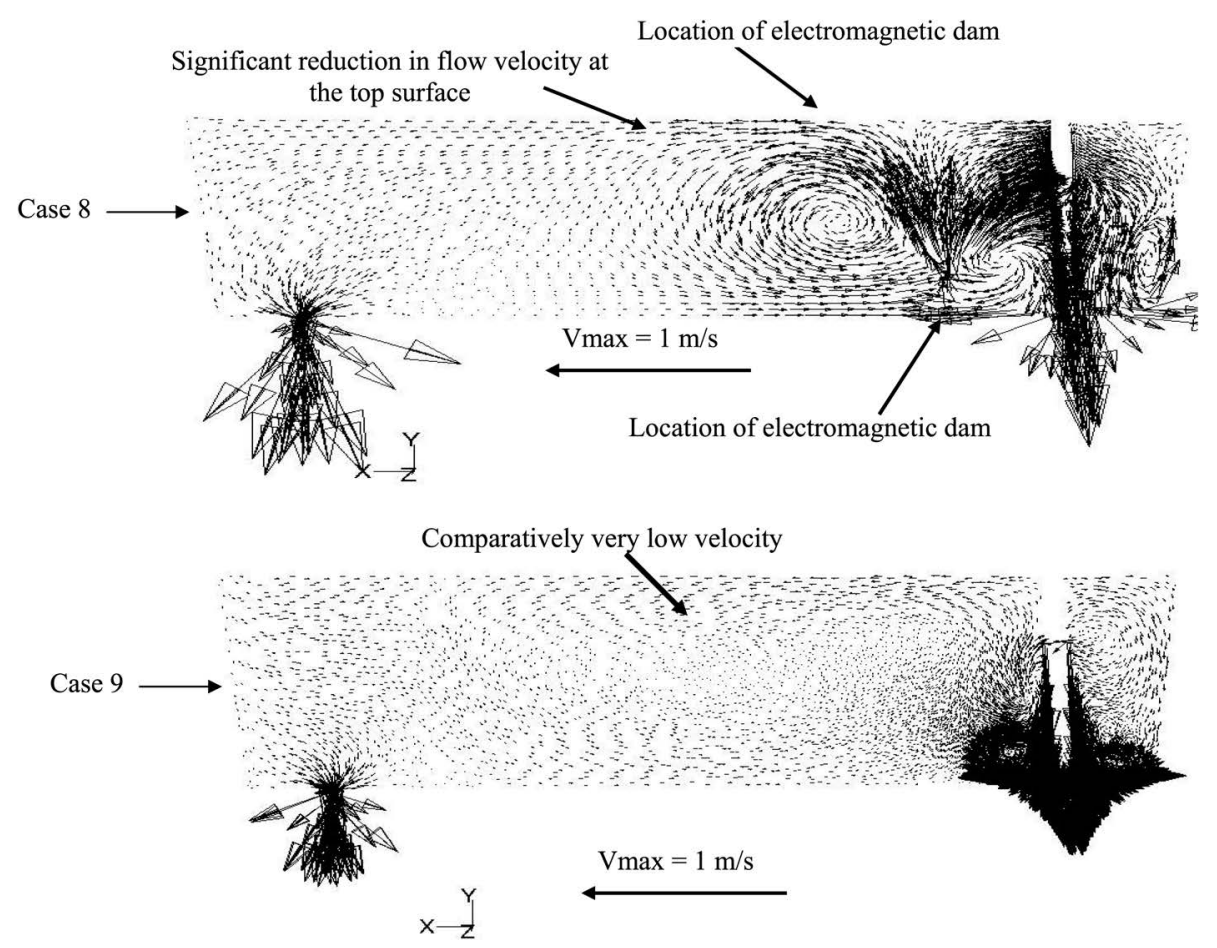

Fig. 9. Flow patterns at symmetrical vertical plane for tundish with three electromagnetic dams of case 8 and tundish with pouring chamber.

domain. However, the flow vectors appear to be more uniform for electromagnetic dam of case 8 as seen from Fig. 9. The rotatory flow near the pouring region can be seen for case 8 in Fig. 9. It can be further seen from Fig. 9 that the flow velocity near the outlet was reduced for case 8 as compared to one observed for case 7 . Figure 10 shows the comparison of flow pattern at vertical $\mathrm{YZ}$ plane $(\mathrm{X}=3.4)$ for case 8 and Case 9. The flow pattern in Fig. 10 for both the cases appears to be similar. However, the circulation cells at the bottom was found to be near the centre for case 9 and it was identified at the corners for case 8 . Thus, the presence of mechanical device (pouring chamber) results in the suppression of flow in the vicinity of pouring stream. The comparison of flow pattern at the meniscus in Fig. 11 was done for further analysis of flow behaviour. The characteristics of the flow pattern at meniscus appear to be common for both the cases. However, some dissimilarity in the flow behaviour can also be seen from Fig. 11. The size of circulation cells at the left corner for pouring chamber was more as compared to one observed for electromagnetic dam. The meniscus velocity also appears to be lower for pouring chamber.

The RTD analysis was required for making any conclusive statement about the performance of electromagnetic dam. Hence, RTD study was performed for case 8 and case 9 presented in Table 1. Figure 12 shows the comparison of RTD curve for both the cases. The shift in peak concentration towards the centre for electromagnetic dam of case 8 as compared to case 9 can be seen from Fig. 12. The fall in peak concentration for case 8 as compared to case 9 can also be noticed from Fig. 12. These differences in the RTD curve indicate the fall in dead volume for electromagnetic dam of case 8 . The RTD characteristics of all the cases can be seen from Table 4. The marginal increase in plug volume and significant drop in dead volume for electromagnetic dam of

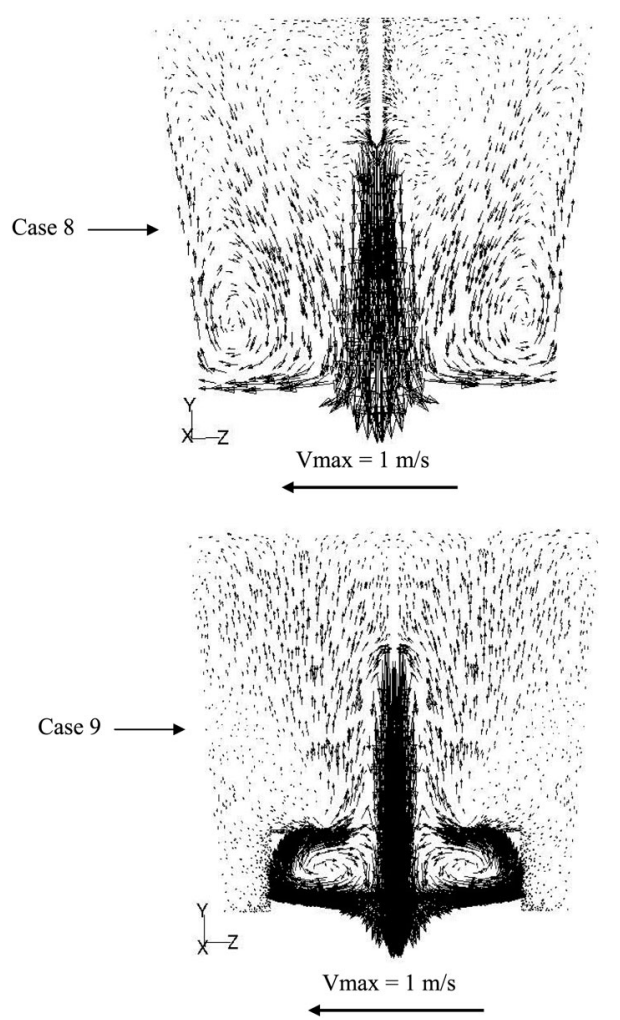

Fig. 10. Flow pattern at vertical $\mathrm{YZ}(\mathrm{X}=3.4)$ plane for tundish with three electromagnetic dams of case 8 and tundish with pouring chamber.

case 8 as compared to case 9 can be noticed from this Table. Thus, pouring chamber might be reducing the velocity below optimum level. The increase in plug to dead volume ratio certifies the superior performance of electromagnetic dam of case 8 over pouring chamber. 

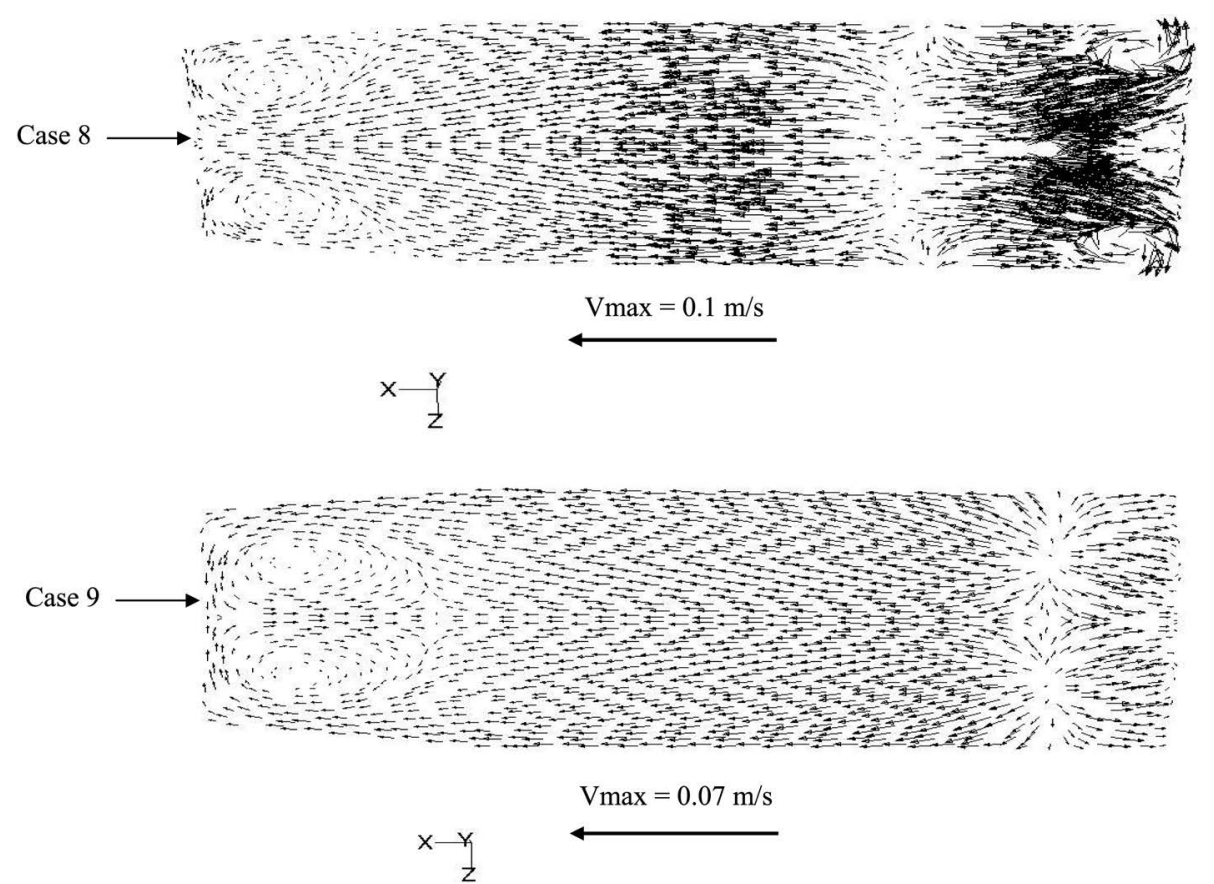

Fig. 11. Flow patterns at meniscus for tundish with three electromagnetic dams of case 8 and tundish with pouring chamber.

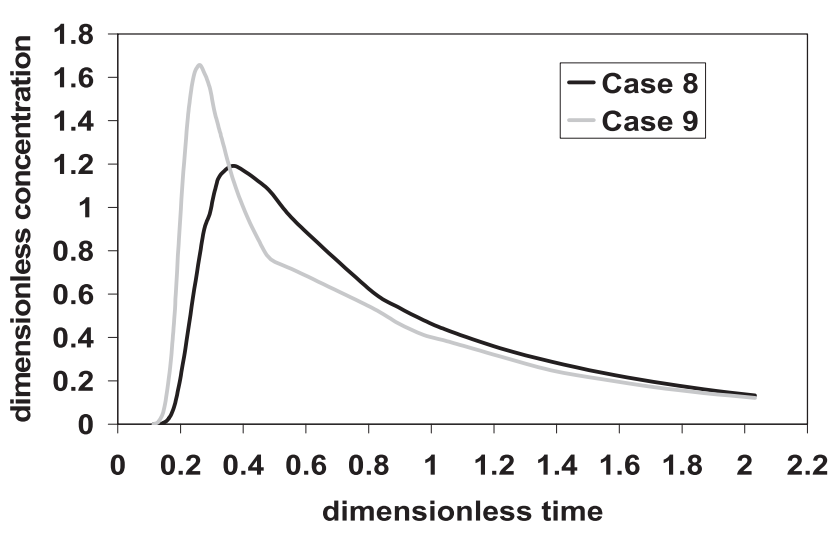

Fig. 12. Comparison of RTD curve for tundish with pouring chamber and tundish with three electromagnetic dams of case 8 .

Table 4. RTD characteristics for case 8 and case 9 .

\begin{tabular}{ccccccc}
\hline S. No. & Cases & $\begin{array}{c}\mathrm{V}_{\mathrm{P}}(\text { Plug } \\
\text { volume } \\
\text { fraction) }\end{array}$ & $\begin{array}{c}\mathrm{V}_{\mathrm{D}} \text { (Dead } \\
\text { volume } \\
\text { fraction) }\end{array}$ & $\begin{array}{c}\mathrm{V}_{\text {Mix }} \\
\text { (Mixed } \\
\text { volume } \\
\text { fraction) }\end{array}$ & $\begin{array}{c}\text { Mean } \\
\text { Residence } \\
\text { Time } \\
(\mathrm{Sec})\end{array}$ & $\begin{array}{c}\text { Ratio of } \\
\text { plug to } \\
\text { dead } \\
\text { volume } \\
\left(\mathrm{V}_{\mathrm{P}} / \mathrm{V}_{\mathrm{D}}\right)\end{array}$ \\
\hline 1 & Case 1 & 0.09 & 0.24 & 0.67 & 472 & 0.38 \\
2 & Case 2 & 0.08 & 0.20 & 0.72 & 498 & 0.40 \\
3 & Case 3 & 0.11 & 0.17 & 0.72 & 516 & 0.65 \\
4 & Case 4 & 0.05 & 0.22 & 0.73 & 485 & 0.23 \\
5 & Case 5 & 0.03 & 0.26 & 0.71 & 460 & 0.12 \\
6 & Case 6 & 0.02 & 0.24 & 0.74 & 473 & 0.08 \\
7 & Case 7 & 0.03 & 0.25 & 0.72 & 467 & 0.12 \\
8 & Case 8 & 0.15 & 0.17 & 0.68 & 516 & 0.88 \\
9 & Case 9 & 0.13 & 0.24 & 0.63 & 472 & 0.54 \\
\hline
\end{tabular}

\section{Conclusion}

The flow control phenomenon driven by electromagnetic forces in the tundish was investigated through numerical modelling. The conclusions are presented:

- Flow control in a tundish can be achieved using electromagnetic forces applied in the form of dam. This type of arrangement was named as electromagnetic dam.

- The flow behaviour generated by a single electromagnetic dam was found to be close to the characteristics of the desired flow pattern. However, the high velocity near the outlet and at the meniscus appears to be the major drawback for this device.

- The flow pattern generated by using a single electromagnetic dam of low magnetic strength appears to be similar to a tundish without flow control device. However, it was found that surface directed flow can be created by increasing the magnetic strength beyond 0.15 tesla.

- The combination of two electromagnetic dams can reduce velocity magnitude generated by a single electromagnetic dam of optimum magnetic strength.

- The reduction of meniscus velocity generated by combination of two electromagnetic dams was required to achieve superior flow pattern as compared to pouring chamber.

- The combination of three electromagn etic dams was proposed to derive the improved fluid flow characteristics as compared to pouring chamber.

- The comparative analysis of fluid flow behaviour and RTD results of tundish shows superior performance for combination of three electromagnetic dams over pouring chamber.

- The combination of electromagnetic dams of optimum magnetic strength can be used as a replacement of mechanical devices, such as pouring chamber. 


\section{Nomenclature}

$\bar{C}$ : Mass fraction of the injected tracer

$C_{a v i}:$ Average concentration of tracer from $i_{\text {th }}$ outlet

$\mathrm{H}$ : Bath height of the tundish

L: Length of half tundish

$L_{\infty}$ : Characteristic length

z: Hartmann number

$\mathrm{Re}_{\mathrm{m}}$ : Magnetic Reynolds number

k: Turbulent kinetic energy

P: Pressure

RTD: Residence time distribution

t: Time

$\mathrm{t}_{\mathrm{m}}$ : Actual mean residence time

$t_{\text {min }}$ : Average breakthrough time

$\mathrm{t}_{\mathrm{r}}$ : Theoretical mean residence time

$\bar{U}$ : Velocity

$U_{\infty}$ : Characteristic velocity

$u^{\prime}$ : Velocity fluctuation

$\mathrm{V}$ : Volume

B: Magnetic Field Strength

Greek Symbols
$\rho$ : Density of steel
$\mu$ : Molecular viscosity of steel
$\mu_{t}$ : Turbulent viscosity of steel
$\mu_{\text {eff: }}$ Effective viscosity of steel
$\sigma_{C}$ : Turbulent Schmidt number

$\varepsilon$ : Rate of dissipation of turbulent kinetic energy

$\tau^{l}$ : Laminar shear stress

$\tau^{t}$ : Turbulent shear stress

$\sigma$ : Electrical conductivity

$\mu_{\infty}$ : Magnetic Permeability

Suffix

$\mathrm{i}, \mathrm{j}, \mathrm{k}$ : Three Cartesian co-ordinate directions $\mathrm{x}, \mathrm{y}$ and $\mathrm{z}$

DV: Dead volume

MV: Mixed volume

PV: Plug Volume

\section{REFERENCES}

1) R. D. Morales, J. Palafox-Ramos, J. Barreto, S. Lopez-Ramirez and D. Zacharias: Metall. Mater. Trans. B, 31 (2000), 1505.

2) A. Tripathi and S. K. Ajmani: ISIJ Int., 45 (2005), 1616.

3) Y. Wang, Y. Zhong, B. Wang, Z. Lei, W. Ren and Z. Reni: ISIJ Int., 49 (2009), 1542.

4) A Tripathi: Appl. Math. Model., 35 (2011), 5075.

5) B. Muck, C. Gunther, U. Muller and L. Buchller: J. Fluid Mech., 418 (2000), 265.

6) D. Lee and H. Choi: J. Fluid Mech., 439 (2001), 367.

7) B. Sreenivasan and T. Alboussiere: J. Fluid Mech., 464 (2002), 287.

8) L. Rossi, J. C. Vassilicos and Y. Hardalupas: J. Fluid Mech., 558 (2006), 207.

9) E. V. Votyakov, Egbertzienicke and Y. Kolesnikov: J. Fluid Mech., $610(2008), 131$.

10) Y. Man, H. G. Lee and S. H. Seong: J. Mater. Proc. Technol., 133 (2003), 322.

11) M. Hughes, K. A. Pericleous and M. Cross: Appl. Math. Model., 18 (1994), 150.

12) T. W. H. Sheu and R. K. Lin: Int. J. Numer. Meth. Fl., 45 (2004), 1209. 